This is an accepted manuscript of an article published by Taylor \& Francis in Integral Transforms and Special Functions on 2018-09-28, available online: https://doi.org/10.1080/10652469.2018.1525369

\title{
Uniform convergent expansions of the Gauss hypergeometric function in terms of elementary functions
}

\author{
Chelo Ferreira ${ }^{1}$, José L. López ${ }^{2}$, and Ester Pérez Sinusía ${ }^{1}$ \\ ${ }^{1}$ Dpto. de Matemática Aplicada, IUMA, Universidad de Zaragoza \\ e-mail: cferrei@unizar.es, ester.perez@unizar.es \\ ${ }^{2}$ Dpto. de Ingeniería Matemática e Informática and INAMAT, Universidad Pública de Navarra \\ e-mail: jl.lopez@unavarra.es
}

\begin{abstract}
We consider the hypergeometric function ${ }_{2} F_{1}(a, b ; c ; z)$ for $z \in \mathbb{C} \backslash[1, \infty)$. For $\Re a \geq 0$, we derive a convergent expansion of ${ }_{2} F_{1}(a, b ; c ; z)$ in terms of the function $(1-z)^{-a}$ and of rational functions of $z$ that is uniformly valid for $z$ in any compact in $\mathbb{C} \backslash[1, \infty)$. When $a \in \mathbb{N}$, the expansion also contains a logarithmic term of the form $\log (1-z)$. For $\Re a \leq 0$, we derive a convergent expansion of $(1-z)^{a}{ }_{2} F_{1}(a, b ; c ; z)$ in terms of the function $(1-z)^{-a}$ and of rational functions of $z$ that is uniformly valid for $z$ in any compact in $\mathbb{C} \backslash[1, \infty)$ in the exterior of the circle $|z-1|=r$ for arbitrary $r>0$. The expansions are accompanied by realistic error bounds. Some numerical experiments show the accuracy of the approximation.
\end{abstract}

2010 AMS Mathematics Subject Classification: 33C05; 41A58; 41A80.

Keywords \& Phrases: hypergeometric function; convergent expansions; uniform expansions.

\section{Introduction}

In literature, we can find a variety of expansions (convergent or not) of the special functions of mathematical physics. These expansions have the important property of being given in terms of elementary functions: mostly, powers or inverse powers of a certain variable $z$ and, sometimes, other elementary functions. However, these expansions are not usually valid simultaneously for

\footnotetext{
*Contact: José L. López, e-mail: jl.lopez@unavarra.es
} 
small and large values of $|z|$. Thus, it would be interesting to derive new convergent expansions in terms of elementary functions that hold uniformly in $z$ in a large region of the complex plane that include small and large values of $|z|$.

In [1] and 2], the authors derived new uniform convergent expansions of the incomplete gamma functions and the Bessel functions respectively in terms of elementary functions. The starting point of the technique used in [1] and [2] is an appropriate integral representation of these functions. The key point is the use of the Taylor expansion, at an appropriate point of the integration interval, of a certain factor of the integrand that is independent of the variable $z$. This fact translates into a convergent uniform expansion in a large region of the complex $z$-plane. The expansions given in [1] and [2] are accompanied by error bounds and numerical experiments showing the accuracy of the approximations.

In this work, we continue that line of investigation considering the Gauss hypergeometric function ${ }_{2} F_{1}(a, b ; c ; z)$. We consider ${ }_{2} F_{1}(a, b ; c ; z)$ as a function of the complex variable $z$, and derive new convergent expansions uniformly valid in an unbounded region of the complex $z$-plane that contains the point $z=0$. The hypergeometric function ${ }_{2} F_{1}(a, b ; c ; z)$ is defined by the Gauss series

$$
{ }_{2} F_{1}(a, b ; c ; z)=\sum_{s=0}^{\infty} \frac{(a)_{s}(b) s}{(c)_{s} s !} z^{s}=\frac{\Gamma(c)}{\Gamma(a) \Gamma(b)} \sum_{s=0}^{\infty} \frac{\Gamma(a+s) \Gamma(b+s)}{\Gamma(c+s) s !} z^{s}
$$

on the disk $|z|<1$, and by analytic continuation elsewhere. In general, ${ }_{2} F_{1}(a, b ; c ; z)$ does not exist when $c=0,-1,-2, \ldots$ The branch obtained by introducing a cut from 1 to $+\infty$ on the real $z$-axis, that is, the branch in the sector $|\operatorname{ph}(1-z)| \leq \pi$, is the principal branch (or principal value) of ${ }_{2} F_{1}(a, b ; c ; z)$.

Our starting point is the Euler integral representation of the hypergeometric function [3, Sec 15.6, Eq. (15.6.1)]

$$
{ }_{2} F_{1}(a, b ; c ; z)=\frac{\Gamma(c)}{\Gamma(b) \Gamma(c-b)} \int_{0}^{1} \frac{t^{b-1}(1-t)^{c-b-1}}{(1-z t)^{a}} d t, \quad \Re c>\Re b>0,
$$

valid for $|\operatorname{ph}(1-z)|<\pi$. For reasons that will become clear later, it is convenient to consider the integral (1.2) only for $\Re a \geq 0$. When $\Re a \leq 0$, we consider instead the integral representation [3. Sec 15.8(i), Eq. (15.8.1)]

$$
{ }_{2} F_{1}(a, b ; c ; z)=\frac{\Gamma(c)(1-z)^{-a}}{\Gamma(b) \Gamma(c-b)} \int_{0}^{1} t^{c-b-1}(1-t)^{b-1}\left(1+\frac{z}{1-z} t\right)^{-a} d t, \quad \Re c>\Re b>0,
$$

valid for $|\operatorname{ph}(1-z)|<\pi$. When $b$ and $c-b$ are positive integers, the Gauss hypergemetric function is an elementary function of $z$.

By a repeated use of the contiguous formulas of the Gauss hypergeometric function [3. Sec. 15.5, eqs. 15.5.11 and 15.518] (the first one with the roles of the parameters $a$ and $b$ interchanged), we have that ${ }_{2} F_{1}(a, b ; c ; z)$, with $\Re b \leq 0$ and/or $\Re c \leq \Re b$, may be written as a linear combination of elementary functions of $a, b, c, z$ and Gauss hypergeometric functions with $\Re c>\Re b>0$. Therefore, without loss of generality, in the remaining of the paper we restrict ourselves to $\Re c>\Re b>0$. 
The power series expansion (1.1) may be derived from (1.2) by replacing the factor $(1-z t)^{-a}$ by its Taylor series at the origin and interchanging series and integral. The Taylor expansion converges for $t \in[0,1]$, but the convergence is not uniform in $|z|$. Therefore, expansion (1.1) is convergent, but not uniformly in $|z|$ as the remainder is unbounded for large $|z|$.

The asymptotic expansion of ${ }_{2} F_{1}(a, b ; c ; z)$ ([3, Sec. 15.2, Eq. (15.2.2)] and [3, Sec. 15.8, Eqs. (15.8.2) and (15.8.8)]) is given by

$$
\begin{aligned}
{ }_{2} F_{1}\left(\begin{array}{c}
a, b \\
c
\end{array} ; z\right)= & \frac{\pi \Gamma(c)}{\sin (\pi(b-a))}\left\{\frac{(-z)^{-a}}{\Gamma(b) \Gamma(c-a) \Gamma(a-b+1)} \sum_{s=0}^{\infty} \frac{(a)_{s}(a-c+1)_{s}}{(a-b+1)_{s} s ! z^{s}}\right. \\
& \left.-\frac{(-z)^{-b}}{\Gamma(a) \Gamma(c-b) \Gamma(b-a+1)} \sum_{s=0}^{\infty} \frac{(b)_{s}(b-c+1)_{s}}{(b-a+1)_{s} s ! z^{s}}\right\},
\end{aligned}
$$

for $b-a \notin \mathbb{N} \cup\{0\}$ and $|\operatorname{ph}(-z)|<\pi$. If $b-a=m \in \mathbb{N} \cup\{0\},|z|>1$ and $|\operatorname{ph}(-z)|<\pi$, we have

$$
\begin{aligned}
& { }_{2} F_{1}\left(\begin{array}{c}
a, a+m \\
c
\end{array} ; z\right)=\frac{\Gamma(c)(-z)^{-a}}{\Gamma(a+m)} \sum_{k=0}^{m-1} \frac{(a)_{k}(m-k-1) !}{k ! \Gamma(c-a-k) z^{k}} \\
& +\frac{\Gamma(c)(-z)^{-a}}{\Gamma(a)} \sum_{k=0}^{\infty} \frac{(-1)^{k}(a+m)_{k}}{k !(k+m) ! \Gamma(c-a-k-m) z^{k+m}} \\
& \times(\log (-z)+\psi(k+1)+\psi(k+m+1) \\
& -\psi(a+k+m)-\psi(c-a-k-m)) \text {, }
\end{aligned}
$$

where $\psi$ denotes the digamma function. Expansions (1.4) and (1.5) are asymptotic for large $|z|$, but the remainders are unbounded for small $|z|$ and then, the expansions are not uniform in $|z|$. These asymptotic expansions may be derived by applying Watson's lemma [4, Chap. 1].

As an illustration of the uniform approximations that we are going to obtain in this paper (see Theorem 1 below), we derive, for example, the following one,

$$
\begin{aligned}
{ }_{2} F_{1}\left(\frac{1}{2}, \frac{29}{10}, \frac{39}{10} ; z\right)= & \frac{29\left(-3465 z^{3}+29260 z^{2}+421344 z-21888\right)}{21000002^{9 / 10} z^{4}} \\
& -\frac{29\left(162329 z^{3}+231724 z^{2}+410400 z-21888\right) \sqrt{1-z}}{21000002^{9 / 10} z^{4}}+\epsilon(z),
\end{aligned}
$$

with $|\epsilon(z)|<0.015$ in the negative half plane $\Re z \leq 0$. When $z=0$, the right hand side of (1.6) must be understood in the limit sense, obtaining $1=0.998491+\epsilon(0)$, and verifying that the absolute value of the error at $z=0$, that is $\epsilon(0)=0.00150906$, is smaller than 0.015 . On the other hand, when we multiply the above formula by $\sqrt{-z}$ and take the limit $z \rightarrow-\infty$, we obtain $1.20833=1.20129+\lim _{z \rightarrow-\infty} \sqrt{-z} \epsilon(z)$, that is, the absolute value of the error in the approximation of $\sqrt{-z}{ }_{2} F_{1}\left(\frac{1}{2}, \frac{29}{10}, \frac{39}{10} ; z\right)$ at $z=-\infty$ is 0.00705 ; a relative error smaller than $0.6 \%$.

In order to derive a uniform convergent expansion of ${ }_{2} F_{1}(a, b ; c ; z)$, we apply the technique proposed in [1] and [2]: we consider a Taylor expansion of the factor $t^{b-1}(1-t)^{c-b-1}$ in (1.2) 
and of the factor $t^{c-b-1}(1-t)^{b-1}$ in (1.3) . These functions are not analytic at the end points of the interval of integration unless $b \in \mathbb{N}$ and $c-b \in \mathbb{N}$. Following the arguments given in [2], we must consider the Taylor expansions of the factors $t^{b-1}(1-t)^{c-b-1}$ and $t^{c-b-1}(1-t)^{b-1}$ at the middle point $t=1 / 2$ of the integration interval $(0,1)$ in the corresponding integrals (1.2) and (1.3), in such a way that we assure that the open interval of integration $(0,1)$ is contained into the disk of convergence of these Taylor series. These Taylor expansions are convergent for any $t$ in the integration interval of (1.2) or (1.3) and, obviously, they are independent of $z$. After the interchange of the series and the integral, the independence with respect to $z$ translates into a remainder that may be bounded independently of $z$ in a large unbounded region of the complex $z$-plane that contains the point $z=0$ and that we specify in Theorems 1 and 2 below. In the following section we consider the integral representation (1.2) for $\Re a \geq 1$. In Section 3 we consider the integral representation (1.3) for $\Re a \leq 1$. Throughout the paper we use the principal $\operatorname{argument} \arg z \in(-\pi, \pi]$.

\section{A uniform convergent expansion of ${ }_{2} F_{1}(a, b ; c ; z)$ for $\Re a \geq 0$}

In this section we consider the integral representation (1.2). We define the extended sector (see Figure 1):

$$
S_{\theta}:=\{\theta \leq|\arg (z)| \leq \pi\} \cup\left\{z \in \mathbb{C} ;\left|z-\frac{1}{2}\right| \leq \frac{1}{2} \text { and }|z-1| \geq \sin \theta\right\},
$$

with arbitrary $0<\theta \leq \pi / 2$. We have the following theorem.

Theorem 1. For $\Re a \geq 0, \Re c>\Re b>0, z \in S_{\theta}$, with $0<\theta \leq \pi / 2$, and $n=1,2,3, \ldots$,

$$
{ }_{2} F_{1}(a, b ; c ; z)=\frac{\Gamma(c)}{\Gamma(b) \Gamma(c-b)} \sum_{k=0}^{n-1} A_{k}(b, c) H_{k}(z, a)+R_{n}(a, b, c ; z),
$$

where the coefficients $A_{k}(b, c)$ are given by

$$
\begin{aligned}
A_{k}(b, c) & :=2^{k+2-c} \sum_{j=0}^{k}(-1)^{j} \frac{(1-b)_{j}(1+b-c)_{k-j}}{j !(k-j) !} \\
& =2^{k+2-c} \frac{(1+b-c)_{k}}{k !}{ }_{2} F_{1}(1-b,-k ; c-b-k ;-1),
\end{aligned}
$$

and $H_{k}(z, a)$ are the elementary functions

$$
H_{k}(z, a):=\frac{(-1)^{k}}{2^{k} z^{k+1}} \sum_{j=0}^{k}\left(\begin{array}{c}
k \\
j
\end{array}\right) 2^{j}(z-2)^{k-j}\left[\frac{1-(1-z)^{j+1-a}}{j+1-a}\left(1-\delta_{j, a-1}\right)-\delta_{j, a-1} \log (1-z)\right]
$$


that may also be represented in terms of hypergeometric functions with parameter $b=1$,

$$
H_{k}(z, a):=\frac{1}{(k+1) 2^{k+1}}\left[(-1)^{k}{ }_{2} F_{1}\left(a, 1 ; k+2 ; \frac{z}{2}\right)+(1-z)^{-a} F_{1}\left(a, 1 ; k+2 ; \frac{z}{2(z-1)}\right)\right] .
$$

The coefficients $A_{k}(b, c)$ and the functions $H_{k}(z, a)$ can be computed recursively in the form

$$
A_{k}(b, c)=\frac{2}{k}\left[(2 b-c) A_{k-1}(b, c)+2(k-c) A_{k-2}(b, c)\right], \quad k \geq 2,
$$

with $A_{0}(b, c)=2^{2-c}, A_{1}(b, c)=(2 b-c) 2^{3-c}$, and for $k=1,2,3, \ldots$ and $a \neq 1$,

$H_{k}(z, a)=\frac{(-1)^{k}-(1-z)^{1-a}}{z(1-a) 2^{k}}+\frac{k}{z(1-a)} H_{k-1}(z, a+1), H_{0}(z, a)=\frac{1}{z(1-a)}\left[1-(1-z)^{1-a}\right]$.

On the other hand, for $k=1,2,3, \ldots$ and $a=1$,

$$
H_{k}(z, 1)=\frac{(-1)^{k}-1}{2^{k} k z}+\left(\frac{1}{z}-\frac{1}{2}\right) H_{k-1}(z, 1), \quad H_{0}(z, 1)=-\frac{1}{z} \log (1-z) .
$$

When $z=0$, the above formulas must be understood in the limit sense. For $n>\Re c-1$, the remainder is bounded in the form

$$
\left|R_{n}(a, b, c ; z)\right| \leq \frac{2 e^{\pi|\Im a|}|\Gamma(c)| \Gamma(1-\Re c+n)}{\pi|\Gamma(b)||\Gamma(c-b)|[\sin (\theta)]^{\Re a}}\left(\frac{|\sin [(c-b) \pi]|}{\Gamma(1-\Re b+n)}+\frac{|\sin (b \pi)|}{\Gamma(1+\Re b-\Re c+n)}\right) .
$$

The remainder term behaves as $R_{n}(z, a, b, c) \sim n^{-\min \{\Re b, \Re c-\Re b\}}$ as $n \rightarrow \infty$ uniformly in $|z|$ in the extended sector $S_{\theta}$.

Proof. Consider the truncated Taylor expansion of the factor $f(t):=t^{b-1}(1-t)^{c-b-1}$ in (1.2) at the middle point $t=1 / 2$ of the integration interval $(0,1)$,

$$
t^{b-1}(1-t)^{c-b-1}=\sum_{k=0}^{n-1} A_{k}(b, c)\left(t-\frac{1}{2}\right)^{k}+r_{n}(b, c, t), \quad t \in(0,1)
$$

where $A_{k}(b, c)$ is defined by (2.3) and $r_{n}(b, c, t)$ is the Taylor remainder

$$
r_{n}(b, c, t):=\sum_{k=n}^{+\infty} A_{k}(b, c)\left(t-\frac{1}{2}\right)^{k}, \quad t \in(0,1) .
$$

Using the differential equation $t(1-t) f^{\prime}=[b-1+(2-c) t] f$ satisfied by the function $f(t)$, we obtain that the Taylor coefficients $A_{k}(b, c)$ can be computed recursively by (2.6).

Replacing (2.10) into the integral representation (1.2) and interchanging sum and integral we obtain (2.2) with

$$
R_{n}(a, b, c ; z):=\frac{\Gamma(c)}{\Gamma(b) \Gamma(c-b)} \sum_{k=n}^{\infty} A_{k}(b, c) H_{k}(z, a)
$$




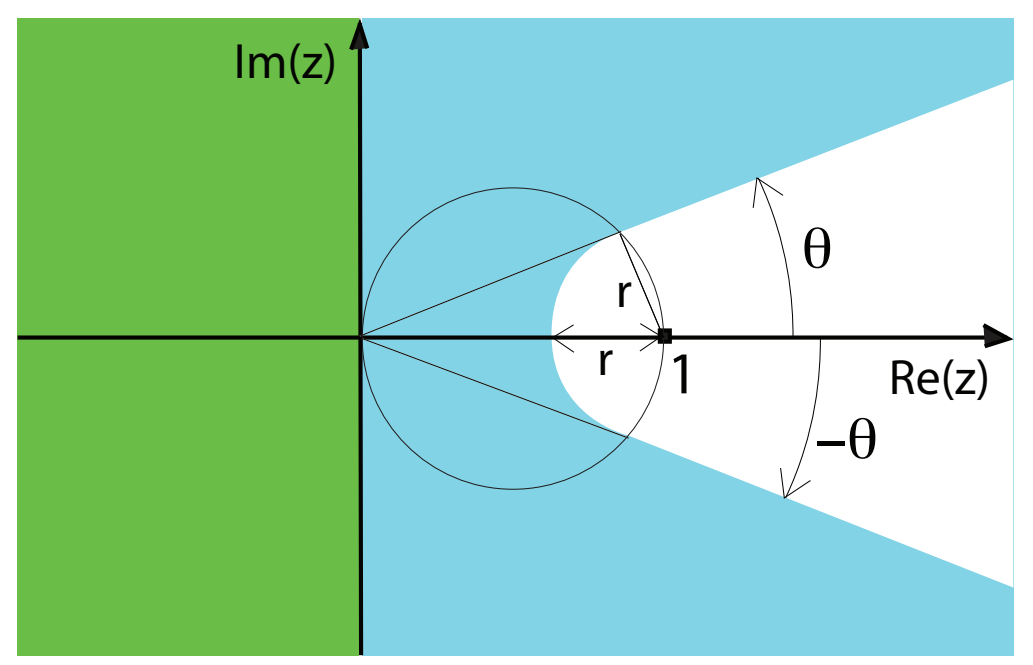

Figure 1: The blue and green regions comprise the extended sector $S_{\theta}$ defined in (2.1), with $r:=\sin \theta$. In particular, $S_{\pi / 2}$ is just the half plane $\Re z \leq 0$ and $\lim _{\theta \rightarrow 0} S_{\theta}=\mathbb{C} \backslash[1, \infty)$. In this region, the remainder $R_{n}(a, b, c ; z)$ is bounded independently of $|z|$ by the right hand side of (2.9) $)$.

$A_{k}(b, c)$ given in (2.3) and

$$
H_{k}(z, a):=\int_{0}^{1}\left(t-\frac{1}{2}\right)^{k}(1-z t)^{-a} d t=\frac{(-1)^{k}}{2^{k} z} \int_{1-z}^{1}\left(1-\frac{2}{z}+2 \frac{u}{z}\right)^{k} u^{-a} d u .
$$

Expanding the first factor of the integrand in the last integral in powers of $u$ and integrating term-wise we obtain (2.4). On the other hand, splitting the first integral at $t=1 / 2$ and integrating the integrals separately we obtain (2.5). The recurrence relations (2.7) and (2.8) follow by integrating by parts in any of the integrals in (2.13).

In order to prove the convergence of (2.2), we observe that, for $t \in[0,1]$, we have that $\mid(1-$ $z t)^{-a} \mid \leq e^{\pi|\Im a|} M(z, a)$, with

$$
M(z, a):= \begin{cases}1, & \text { if } \Re(z) \leq 0 \\ |1-z|^{-\Re a}, & \text { if } \Re(1 / z) \geq 1 \\ |\sin (\arg (z))|^{-\Re a}, & \text { if } 0<\Re(1 / z)<1 .\end{cases}
$$

The regions of the complex $z$-plane considered in this formula are depicted in Figure 2. Using this bound in the first integral in (2.13) we find that

$$
\left|H_{k}(z, a)\right| \leq \int_{0}^{1}\left|t-\frac{1}{2}\right|^{k}\left|(1-z t)^{-a}\right| d t \leq \frac{e^{\pi|\Im a|} M(z, a)}{2^{k}(k+1)} .
$$

On the other hand, for $z \in S_{\theta}$, we have that $M(z, a) \leq[\sin (\theta)]^{-\Re a}$. This inequality may be proved using the following geometrical arguments: (i) at the points of the circle $\mid z-$ $1 / 2 \mid=1 / 2$ we have that $|1-z|=|\sin (\arg (z))|$; (ii) the closest points of the sector $\theta \leq$ 


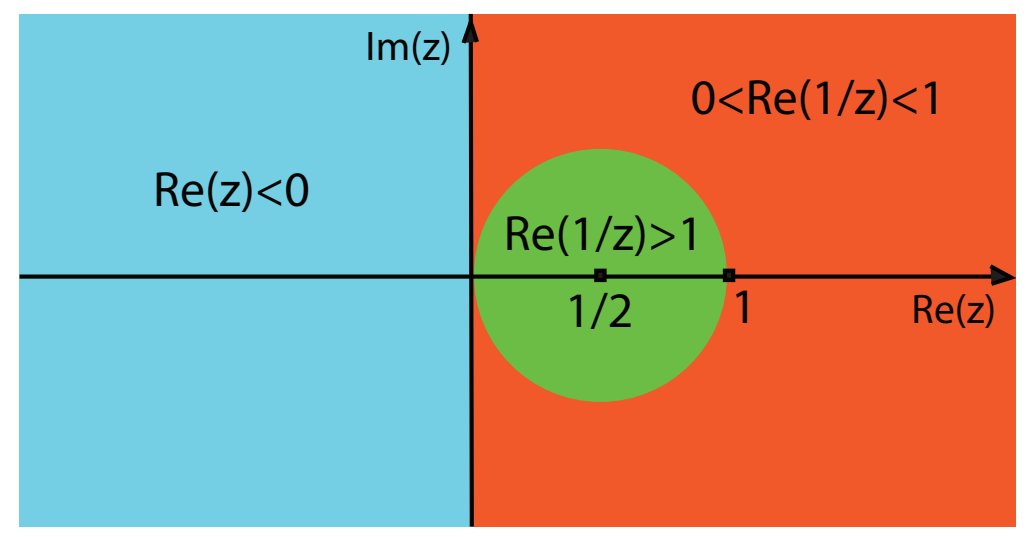

Figure 2: Different regions considered in formula (2.14). The green region $\Re(1 / z)>1$ is the open disk of radius $1 / 2$ with center at $z=1 / 2$. The red region $0<\Re(1 / z)<1$ is the intersection of the half plane $\Re z>0$ with the exterior to this disk.

$|\arg (z)|<\pi / 2$ to the point $z=1$ are just the two points obtained from the intersection of the rays $\arg z= \pm \theta$ with the circle $|z-1 / 2|=1 / 2$; (iii) the closest points of the region $\left\{z \in \mathbb{C} ;\left|z-\frac{1}{2}\right| \leq \frac{1}{2}\right.$ and $\left.|z-1| \geq \sin \theta\right\}$ to the point $z=1$ are those of the portion of circle $|z-1|=\sin \theta$ contained inside this region.

On the other hand, from [5, eqs. (16) and (25)] and the integral representation of ${ }_{2} F_{1}[3$, Sec. 15.6, eq. 15.6.1], we find that, for $k+2>\Re c$, the coefficients $A_{k}(b, c)$ may be written in the form

$$
\begin{aligned}
A_{k}(b, c)= & \frac{\sin [(c-b) \pi]}{\pi} \int_{0}^{1} t^{k+1-c}(1-t)^{c-b-1}(1-t / 2)^{-k-1} d t \\
& +\frac{(-1)^{k} \sin (b \pi)}{\pi} \int_{0}^{1} t^{k+1-c}(1-t)^{b-1}(1-t / 2)^{-k-1} d t .
\end{aligned}
$$

Taking into account that $(1-t / 2)^{-k-1} \leq 2^{k+1}$ for $t \in(0,1)$, we have the following bound for coefficients $A_{k}(b, c)$ when $n+2>\Re c$,

$$
\left|A_{k}(b, c)\right| \leq \frac{2^{k+1}}{\pi}\left(|\sin [(c-b) \pi]| \frac{\Gamma(\Re c-\Re b) \Gamma(2-\Re c+k)}{\Gamma(2-\Re b+k)}+|\sin (b \pi)| \frac{\Gamma(\Re b) \Gamma(2-\Re c+k)}{\Gamma(2+\Re b-\Re c+k)}\right) .
$$

Using (2.15) and (2.16) in (2.12) we find

$$
\begin{aligned}
\left|R_{n}(a, b, c ; z)\right| \leq & \frac{2 e^{\pi|\Im a|}|\Gamma(c)| \sin [(c-b) \pi] \mid \Gamma(\Re c-\Re b)}{\pi|\Gamma(b)||\Gamma(c-b)|[\sin (\theta)]^{\Re a}} \sum_{k=n}^{+\infty} \frac{\Gamma(2-\Re c+k)}{(k+1) \Gamma(2-\Re b+k)} \\
& +\frac{2 e^{\pi|\Im a|}|\Gamma(c)||\sin (b \pi)| \Gamma(\Re b)}{\pi|\Gamma(b)||\Gamma(c-b)|[\sin (\theta)]^{\Re a}} \sum_{k=n}^{+\infty} \frac{\Gamma(2-\Re c+k)}{(k+1) \Gamma(2+\Re b-\Re c+k)} .
\end{aligned}
$$


For $1-\Re c+k>0$ we have that

$$
\frac{\Gamma(2-\Re c+k)}{k+1}<\frac{\Gamma(2-\Re c+k)}{k+1-\Re c}=\Gamma(1-\Re c+k),
$$

and then (2.17) may be bounded in the form

$$
\begin{aligned}
\left|R_{n}(a, b, c ; z)\right| \leq \frac{2 e^{\pi|\Im a|}|\Gamma(c)||\sin (c-b) \pi| \Gamma(\Re c-\Re b)}{\pi|\Gamma(b)||\Gamma(c-b)|[\sin (\theta)]^{\Re a}} \frac{\Gamma(1-\Re c+n)}{\Gamma(2-\Re b+n)}{ }_{2} F_{1}\left(\begin{array}{c}
1,1-\Re c+n \mid \\
2-\Re b+n
\end{array}\right) \\
\quad+\frac{2 e^{\pi|\Im a|}|\Gamma(c)||\sin (b \pi)| \Gamma(\Re b)}{\pi|\Gamma(b)||\Gamma(c-b)|[\sin (\theta)]^{\Re a}} \frac{\Gamma(1-\Re c+n)}{\Gamma(2+\Re b-\Re c+n)}{ }_{2} F_{1}\left(\begin{array}{c}
1,1-\Re b+n \\
2+\Re a-\Re b+n \mid
\end{array}\right) .
\end{aligned}
$$

Finally, using formula [3, Sec. 15.4, eq. 15.4.20] for argument unity of ${ }_{2} F_{1}$, we find (2.9).

From the right hand side of (2.9) and the Stirling formula for the gamma function, we find that $R_{n}(a, b, c ; z) \sim n^{-\min \{\Re b, \Re c-\Re b\}}$ when $n \rightarrow+\infty$. Then, the series (2.2) is convergent for $\Re a \geq 0$ and $\Re c>\Re b>0$ and the bound (2.9) shows the uniform character of the expansion (2.2) in the extended sector $S_{\theta}$.

From the differential equation $t(1-t) f^{\prime}=[b-1+(2-c) t] f$ satisfied by the function $f(t)$ used in the proof of the above theorem, it is possible to find, for $\Re b>1 / 2$ and $\Re(c-b)>1 / 2$, a more accurate error bound than (2.9). It is given in the following proposition.

Proposition 1. In the sector $S_{\theta}, 0<\theta \leq \pi / 2$, and for $\Re a \geq 0,2 n \geq \Re b>1 / 2$ and $2 n \geq \Re(c-b)>1 / 2$, the remainder $R_{n}(a, b, c ; z)$ of the expansion (2.2) is bounded in the form

$$
\begin{aligned}
\left|R_{n}(a, b, c, z)\right| \leq & \frac{e^{\pi|\Im a|}}{2^{n}(n+1)[\sin (\theta)]^{\Re a}}\left\{\frac{n\left|A_{n}(b, c)\right|}{4}\left[C_{n}(b)+C_{n}(c-b)\right]\right. \\
& \left.+|n+1-c|\left|A_{n-1}(b, c)\right|\left[C_{n+1}(b)+C_{n+1}(c-b)\right]\right\}
\end{aligned}
$$

where

$$
C_{n}(b):= \begin{cases}\frac{1}{\Re b-1}\left[1-\frac{n !}{(\Re b)_{n}}\right] & \text { if } \quad \Re b \neq 1, \\ H_{n} & \text { if } \quad \Re b=1,\end{cases}
$$

and $H_{n}$ is the harmonic number of order $n$.

Proof. From the differential equation $t(1-t) f^{\prime}=[b-1+(2-c) t] f$ satisfied by the function $f(t)=t^{b-1}(1-t)^{c-b-1}$ and the recurrence relation (2.6) satisfied by the Taylor coefficients of $f(t)$ at $t=1 / 2($ see (2.10) $)$, we find that the Taylor remainder $r_{n}(t) \equiv r_{n}(b, c, t)$ in formula (2.10) is a solution of the boundary value problem

$$
\left\{\begin{array}{l}
t(1-t) r_{n}^{\prime}+[1-b+(c-2) t] r_{n}=\frac{n}{4} A_{n}(b, c)\left(t-\frac{1}{2}\right)^{n-1}+(n+1-c) A_{n-1}(b, c)\left(t-\frac{1}{2}\right)^{n}, \\
r_{n}\left(\frac{1}{2}\right)=0 .
\end{array}\right.
$$


The unique solution of this problem is

$$
r_{n}(t)=t^{b-1}(1-t)^{c-b-1}\left[\frac{n}{4} A_{n}(b, c) B_{n-1}(b, c)+(n+1-c) A_{n-1}(b, c) B_{n}(b, c)\right],
$$

with

$$
B_{n}(b, c):=\int_{1 / 2}^{t} u^{-b}(1-u)^{b-c}\left(u-\frac{1}{2}\right)^{n} d u .
$$

For $2 n \geq \Re b>0$ and $2 n \geq \Re(c-b)>0$, we have that $(u-1 / 2)^{n} u^{-\Re b} \leq(t-1 / 2)^{n} t^{-\Re b}$ for $1 / 2 \leq u \leq t \leq 1$ and $(1 / 2-u)^{n}(1-u)^{\Re(b-c)} \leq(1 / 2-t)^{n}(1-t)^{\Re(b-c)}$ for $0 \leq t \leq u \leq 1 / 2$.

Using these bounds in the definition of $B_{n}(b, c)$ we find that, for $t>1 / 2$,

$$
\left|B_{n}(b, c)\right| \leq\left(t-\frac{1}{2}\right)^{n} t^{-\Re b} \begin{cases}\frac{(1-t)^{\Re(b+1-c)}-2^{\Re(c-b-1)}}{\Re(c-b-1)} & \text { for } c-b \neq 1 \\ -\log [2(1-t)] & \text { for } c-b=1\end{cases}
$$

and, for $t<1 / 2$,

$$
\left|B_{n}(b, c)\right| \leq\left(\frac{1}{2}-t\right)^{n} t^{\Re(b-c)} \begin{cases}\frac{t^{\Re(1-b)}-2^{\Re(b-1)}}{\Re(b-1)} & \text { for } b \neq 1 \\ -\log (2 t) & \text { for } b=1\end{cases}
$$

Therefore, introducing these bounds into the integral definition of the remainder $R_{n}(a, b, c ; z)$,

$$
R_{n}(a, b, c ; z)=\frac{\Gamma(c)}{\Gamma(b) \Gamma(c-b)} \int_{0}^{1} r_{n}(t)(1-z t)^{-a} d t
$$

and using also the bound $\left|(1-z t)^{-a}\right| \leq e^{\pi|\Im a|}[\sin (\theta)]^{-\Re a}$ derived in the proof of Theorem 1, we find the bound (2.19) $-(2.20)$.

Formula (1.6) is the particular case of formulas (2.2) and (2.19) for the specified values of the parameters $a, b$ and $c$ and $n=4$.

In Table 1 we show the first terms of the expansion on the right hand side of (2.2) for $\Re a \geq 0$ and $a \notin \mathbb{N}$. These terms are rational functions of $z$ and functions of $(1-z)^{-a}$. When $a \in \mathbb{N}$, the expansion (2.2) also contains the term $\log (1-z)$.

In Figure 3 we plot ${ }_{2} F_{1}(0.5,1.3 ; 2.5 ; z)$ and the approximations given in Theorem 1 for $n=2,4$ and 6 . These plots show the uniform character of the expansion (2.2).

\section{A uniform convergent expansion of ${ }_{2} F_{1}(a, b ; c ; z)$ for $\Re a \leq 0$}

In this section we consider the integral representation (1.3). For any $0<r \leq 1$, consider the punctured complex plane at $z=1$ with the interval $[1, \infty)$ removed:

$$
C_{r}:=\{z \in \mathbb{C} ;|z-1| \geq r,|\arg (1-z)|<\pi\} .
$$

We have the following theorem. 


\begin{tabular}{|c|c|}
\hline$n$ & $A_{n}(b, c) H_{n}(z, a)$ \\
\hline 1 & $\frac{2^{2-c}\left(1-(1-z)^{1-a}\right)}{(1-a) z}$ \\
\hline 2 & $\frac{2^{2-c}\left(1-(1-z)^{1-a}\right)}{(1-a) z}-\frac{2^{2-c}(2 b-c)\left((-2+a)\left(-1-(1-z)^{1-a}\right)-\frac{2\left(1-(1-z)^{2-a}\right)}{z}\right)}{(1-a)(2-a) z}$ \\
\hline 3 & $\begin{array}{c}\frac{2^{2-c}\left(1-(1-z)^{1-a}\right)}{(1-a) z}+\frac{2^{2-c}\left(2-c+(-2 b+c)^{2}\right)}{(1-a)(2-a)(3-a) z} \\
\times\left(\frac{1}{2}(-3+a)(-2+a)\left(1-(1-z)^{1-a}\right)+\frac{4\left(1-(1-z)^{3-a}\right)}{z^{2}}-\frac{2(-3+a)\left(-1-(1-z)^{2-a}\right)}{z}\right) \\
-\frac{2^{2-c}(2 b-c)\left((-2+a)\left(-1-(1-z)^{1-a}\right)-\frac{2\left(1-(1-z)^{2-a}\right)}{z}\right)}{(1-a)(2-a) z}\end{array}$ \\
\hline
\end{tabular}

Table 1: First few terms in the expansion (2.2) of $\frac{\Gamma(b) \Gamma(c-b)}{\Gamma(c)}{ }_{2} F_{1}(a, b ; c ; z)$ when $\Re a \geq 0$ and $a \notin \mathbb{N}$.

Theorem 2. For $\Re a \leq 0, \Re c>\Re b>0, z \in C_{r}$ with $0<r \leq 1$, and $n=1,2,3, \ldots$,

$$
(1-z)_{2}^{a} F_{1}(a, b ; c ; z)=\frac{\Gamma(c)}{\Gamma(b) \Gamma(c-b)} \sum_{k=0}^{n-1} \tilde{A}_{k}(b, c) \tilde{H}_{k}(z, a)+R_{n}(a, b, c ; z),
$$

where the coefficients $\tilde{A}_{k}(b, c)$ are given by

$$
\begin{aligned}
\tilde{A}_{k}(b, c):= & 2^{k+2-c} \sum_{j=0}^{k}(-1)^{j} \frac{(1+b-c)_{j}(1-b)_{k-j}}{j !(k-j) !} \\
& =2^{k+2-c} \frac{(1-b)_{k}}{k !}{ }_{2} F_{1}(1+b-c,-k ; b-k ;-1),
\end{aligned}
$$

and $\tilde{H}_{k}(z, a)$ are the elementary functions

$$
\tilde{H}_{k}(z, a):=\frac{1}{z^{k+1}} \sum_{j=0}^{k}\left(\begin{array}{c}
k \\
j
\end{array}\right) 2^{j-k}(z-2)^{k-j} \frac{(1-z)^{a}-(1-z)^{j+1}}{j+1-a} .
$$

The coefficients $\tilde{A}_{k}(b, c)$ and functions $\tilde{H}_{k}(z, a)$ can be computed recusively in the form

$$
\tilde{A}_{k}(b, c)=\frac{2}{k}\left[(c-2 b) \tilde{A}_{k-1}(b, c)+2(k-c) \tilde{A}_{k-2}(b, c)\right], \quad k \geq 2,
$$

with $\tilde{A}_{0}(b, c)=2^{2-c}$ and $\tilde{A}_{1}(b, c)=(c-2 b) 2^{3-c}$, and for $k=1,2,3, \ldots$

$$
\tilde{H}_{k}(z, a)=\frac{1-z}{2^{k}(1-a) z}\left[\frac{1}{(1-z)^{1-a}}-(-1)^{k}\right]-\frac{k(1-z)}{(1-a) z} \tilde{H}_{k-1}(z, a-1),
$$



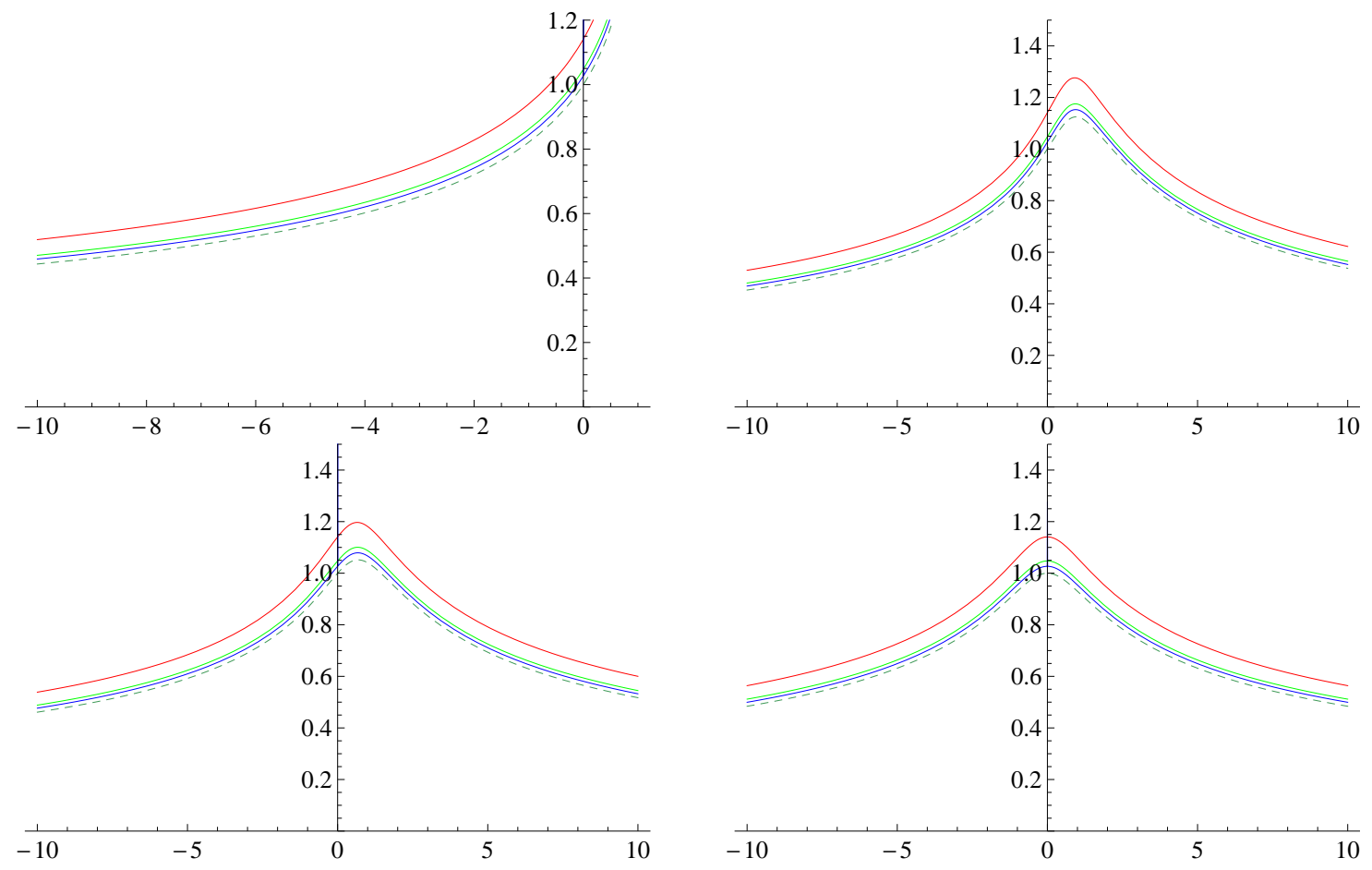

Figure 3: Plots of the absolute value of ${ }_{2} F_{1}(0.5,1.3,2.5, z)$ (dashed) and the approximations given in Theorem 1 for $n=2$ (red), $n=4$ (green) and $n=6$ (blue) in several intervals: [-10,1] (top left), $\left[-10 e^{i \pi / 4}, 10 e^{i \pi / 4}\right]$ (top right), $\left[-10 e^{i \pi / 2}, 10 e^{i \pi / 2}\right]$ (bottom left) and $\left[-10 e^{-i \pi / 3}, 10 e^{-i \pi / 3}\right]$ (bottom right).

with $\tilde{H}_{0}(z, b)=\frac{1-z}{(1-a) z}\left[\frac{1}{(1-z)^{1-a}}-1\right]$.

When $z=0$, the above formulas must be understood in the limit sense.

For any $z \in C_{r}$, and $n>\Re c-2$, the remainder is bounded in the form

$$
\left|R_{n}(a, b, c ; z)\right| \leq \frac{2 e^{\pi|\Im a|}|\Gamma(c)| \Gamma(1-\Re c+n)}{\pi|\Gamma(b)||\Gamma(c-b)| r^{-\Re a}}\left(\frac{|\sin [(c-b) \pi]|}{\Gamma(1-\Re b+n)}+\frac{|\sin (b \pi)|}{\Gamma(1+\Re b-\Re c+n)}\right) .
$$

The remainder term behaves as $R_{n}(a, b, c ; z) \sim n^{-\min \{\Re b, \Re c-\Re b\}}$ as $n \rightarrow \infty$ uniformly for $z \in C_{r}$.

Proof. It is similar to the proof of Theorem 1, but considering the integral representation (1.3) instead of (1.2). That is, we must consider the Taylor expansion of the factor $t^{c-b-1}(1-t)^{b-1}$ at $t=1 / 2$ instead of the expansion of the factor $t^{b-1}(1-t)^{c-b-1}$. We must also replace $z$ by $z /(z-1)$ in the factor $(1-z t)^{-a}$. Then, we only give here a few significant details.

Replacing the truncated series Taylor expansion of $t^{c-b-1}(1-t)^{b-1}$ at $t=1 / 2$ on the right hand side of (1.3) we obtain (3.2) with

$$
R_{n}(a, b, c ; z):=\frac{\Gamma(c)}{\Gamma(b) \Gamma(c-b)} \sum_{k=n}^{\infty} \tilde{A}_{k}(b, c) \tilde{H}_{k}(z, a)
$$


and

$$
\tilde{H}_{k}(z, a):=\int_{0}^{1}\left(t-\frac{1}{2}\right)^{k}\left(1+\frac{z}{1-z} t\right)^{-a} d t=\frac{1-z}{z} \int_{1}^{(1-z)^{-1}}\left(\frac{z-2}{2 z}+\frac{1-z}{z} u\right)^{k} u^{-a} d u .
$$

Expanding the first factor of the integrand in the second integral in powers of $u$ and integrating term-wise we obtain (3.4). Then, we obtain (3.2) with $R_{n}(a, b, c ; z)$ given in (3.7). Now, in order to derive the bound (3.6), instead of a bound for the factor $(1-z t)^{-a}$ valid for every $t \in[0,1]$, we need a bound for the factor $\left(1-z(z-1)^{-1} t\right)^{-a}$ valid for every $t \in[0,1]$. It is given by $\left|\left(1-z(z-1)^{-1} t\right)^{-a}\right| \leq e^{\pi|\Im a|} \bar{M}(z, a)$, with

$$
\bar{M}(z, a):=\max \left\{1,|1-z|^{\Re a}\right\} .
$$

It is clear that $\bar{M}(z, a) \leq r^{\Re a}$ for $z \in C_{r}$ and then, instead of (2.9) we obtain (3.6).

The following proposition is an immediate consequence of Proposition 1 and the proof of Theorem 2.

Proposition 2. In the punctured plane $C_{r}$, with $0<r \leq 1$, and for $\Re a \leq 0,2 n \geq \Re b>1 / 2$ and $2 n \geq \Re(c-b)>1 / 2$, the remainder $R_{n}(a, b, c ; z)$ of the expansion (3.2) is bounded in the form

$$
\begin{aligned}
\left|R_{n}(a, b, c, z)\right| \leq & \frac{e^{\pi|\Im a|} r^{\Re a}}{2^{n}(n+1)}\left\{\frac{n\left|A_{n}(b, c)\right|}{4}\left[C_{n}(b)+C_{n}(c-b)\right]\right. \\
& \left.+|n+1-c|\left|A_{n-1}(c-b)\right|\left[C_{n+1}(b)+C_{n+1}(c-b)\right]\right\},
\end{aligned}
$$

with $C_{n}(b)$ given in (2.20).

In Table 2 we show the first terms of the expansion (3.2) of $\frac{\Gamma(b) \Gamma(c-b)}{\Gamma(c)}(1-z)^{a}{ }_{2} F_{1}(a, b ; c ; z)$. These terms are rational functions of $z$ and functions of $(1-z)^{a}$.

In Figure 4 we plot $(1-z){ }_{2}^{1.5} F_{1}(-1.5,1.7 ; 3.2 ; z)$ and the approximations given in Theorem 2 for $n=2,4$ and 6 . These plots show the uniform character of the expansion (3.2).

\section{Acknowledgments}

This research was supported by the Spanish Ministry of Economía y Competitividad, project MTM2017-83490-P.

\section{Disclosure statement}

No potential conflict of interest was reported by the authors. 


\begin{tabular}{|l|c|}
\hline$n$ & $\tilde{A}_{n}(b, c) \tilde{H}_{n}(z, a)$ \\
\hline 1 & $\frac{2^{2-c}\left((1-z)^{-1+a}-1\right)(1-z)}{(1-a) z}$ \\
\hline 2 & $\frac{2^{2-c}\left(-1+(1-z)^{a}+z\right)}{(1-a) z}+\frac{2^{2-c}(-2 b+c)\left(-(1-z)^{a}(2+(-2+a) z)+(-1+z)(-2+a z)\right)}{(1-a)(2-a) z^{2}}$ \\
\hline 3 & $+\frac{2^{3-c}\left(-\left(2+4 b^{2}+c^{2}-2 b(1+2 c)\right)(1-z)^{a}-\left(2+4 b^{2}+b(2-4 c)+(-2+c) c\right)(-1+z)^{2}\right)}{(1-a)(2-a) z^{2}}$ \\
& $+\frac{2^{4-c}\left(2-c+(-2 b+c)^{2}\right)\left((1-z)^{a}+(-1+z)^{3}\right)}{(1-a)(2-a)(3-a) z^{3}}$ \\
\hline
\end{tabular}

Table 2: First few terms in the expansion (3.2) of $\frac{\Gamma(b) \Gamma(c-b)}{\Gamma(c)}(1-z)^{a}{ }_{2} F_{1}(a, b ; c ; z)$ when $\Re a \leq 0$.
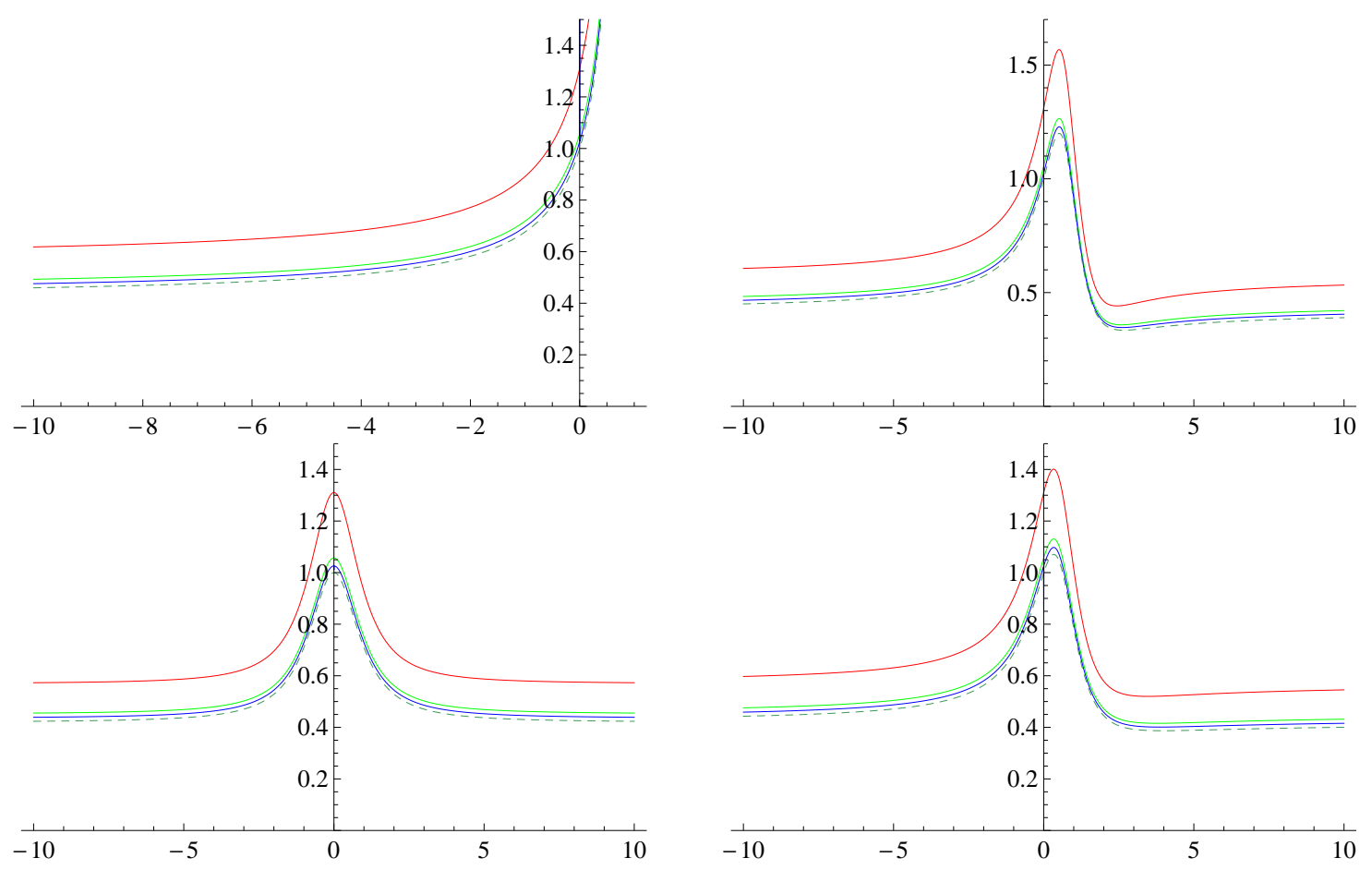

Figure 4: Plots of the absolute value of $(1-z){ }^{1.5}{ }_{2} F_{1}(-1.5,1.7,3.2, z)$ (dashed) and the approximations given in Theorem 2 for $n=2$ (red), $n=4$ (green) and $n=6$ (blue) in several intervals: [-10,1] (top left), $\left[-10 e^{i \pi / 4}, 10 e^{i \pi / 4}\right]$ (top right), $\left[-10 e^{i \pi / 2}, 10 e^{i \pi / 2}\right]$ (bottom left) and $\left[-10 e^{-i \pi / 3}, 10 e^{-i \pi / 3}\right]$ (bottom right). 


\section{References}

[1] Bujanda B, López JL, Pagola PJ. Convergent expansions of the incomplete gamma functions in terms of elementary functions. Anal Appl. 2018;16(3):435-448.

[2] López JL. Convergent expansions of the Bessel functions in terms of elementary functions. Adv Comput Math. 2018;44(1):277-294.

[3] Olde Daalhuis AB. Hypergeometric Function. In: Olver FWJ, Lozier DW, Boisvert RF, Clark CW, editors. NIST Handbook of Mathematical Functions. Cambridge (MA): Cambridge University Press; 2010, p. 383402.

[4] Wong R. Asymptotic approximations of integrals. New York(NY): Academic Press; 1989.

[5] Temme NM. Large parameter cases of the Gauss hypergeometric function. J Comput Math. 2003;153:441462. 